\title{
LAS METODOLOGÍAS BIOGRÁFICAS Y NARRATIVAS Y SUS APORTES PARA EL DESARROLLO DE PROCESOS ANTROPOLÓGICOS ALREDEDOR DE LA SALUD Y LA ENFERMEDAD EN COMUNIDADES CAMPESINAS
}

\author{
Mauricio Múnera Gómez (UdeA)* \\ https://orcid.org/0000-0003-2300-9617
}

\section{RESUMEN}

Este texto presenta algunas reflexiones acerca del uso de las metodologías biográficas y narrativas, para el desarrollo de procesos de investigación alrededor de la salud y la enfermedad en comunidades campesinas del Norte de Antioquia. A partir de la pregunta por cómo el campesino se hace sanador, se da cuenta del aporte de las historias de vida como herramientas que permiten la comprensión de diferentes trayectorias y experiencias de campesinos, que llevan a cabo tareas de salud comunitaria. En sintonía con las metodologías que se abordan en el artículo, se presentan algunas estrategias para la recolección de información: círculos de palabra, observación de prácticas y relatos de experiencias. Así, se destacan las prácticas para el cuidado de la vida de la vida que desarrollan los campesinos y, además, las vías de iniciación de ellos como sanadores. Por último, el artículo reconoce la importancia de las metodologías biográficas y narrativas para la transformación de los modos de investigar y para la consolidación de otras posibilidades de enunciación.

Palabras clave: Metodologías biográficas y narrativas. Historias de vida. Campesinos. Prácticas para el cuidado de la vida.

\section{RESUMO}

\section{METODOLOGIAS BIOGRÁFICAS E NARRATIVAS E SUAS CONTRIBUIÇÕES PARA O DESENVOLVIMENTO DE PROCESSOS ANTROPOLOGICOS EM TORNO DA SAÚDE E DA DOENÇA NAS COMUNIDADES RURAIS}

Este texto apresenta algumas reflexões sobre o uso de metodologias biográficas

\footnotetext{
Doctor en Ciencias Humanas y Sociales de la Universidad Nacional de Colombia, Sede Medellín. Profesor de la Facultad de Educación de la Universidad de Antioquia, Colombia. Integrante del Grupo de Investigación Somos Palabra: formación y contextos. Correo electrónico: mauricio.munera1@udea.edu.co
} 
e narrativas para o desenvolvimento de processos de pesquisa em saúde e doença em comunidades rurais do norte de Antioquia. A partir da questão de como o camponês se torna um curandeiro, é apresentada a contribuição das histórias de vida como ferramentas que permitem a compreensão de diferentes trajetórias e experiências de camponeses que realizam tarefas de saúde comunitária. De acordo com as metodologias abordadas no artigo, são expostas algumas estratégias para coleta de informações: círculos de palavras, observação de práticas e histórias de experiências. Assim, destacamse as práticas de cuidado da vida que os camponeses desenvolvem e, além disso, os modos de iniciação como curandeiros. Por fim, o artigo reconhece a importância das metodologias biográficas e narrativas para a transformação de formas de investigação e para a consolidação de outras possibilidades de enunciação.

Palavras-chave: Metodologias biográficas e narrativas. Histórias de vida. Camponeses. Práticas de cuidado da vida.

\section{ABSTRACT}

\section{BIOGRAPHICAL AND NARRATIVE METHODOLOGIES AND THEIR CONTRIBUTIONS TO THE DEVELOPMENT OF ANTHROPOLOGICAL PROCESSES AROUND HEALTH AND SICKNESS IN RURAL COMMUNITIES}

This text presents some reflections about the use of biographical and narrative methodologies for developing researches around health and sickness in rural communities in North of Antioquia, Colombia. From the question how the peasant becomes in healer, it is possible to recognize the contribution of life stories as tools that allow the understanding of different trajectories and experiences of peasants, who carry out community health tasks. Based on the biographical and narrative methodologies, the text develops some strategies for information gathering: word circles, observation of practices, and stories of experiences. Thus, here are shown the practices for the care of the life that peasants develop and, in addition, the ways of initiating them as healers. Finally, the article recognizes the importance of biographical and narrative methodologies for the transformation of ways to research and to consolidate of other enunciation possibilities.

Keywords: Biographical and narrative methodologies. Life stories. Peasants. Life care practices. 


\section{La investigación como viaje ${ }^{1}$}

La vida es un gran viaje que se compone, a su vez, de migraciones, desplazamientos y retornos. Como viaje, nos impela a estar caminando, pues es el caminar la vía para aprender y enriquecer aquel baúl con el que llegamos a esta tierra. En tanto nos ponemos en camino, comenzamos a reunir experiencias que nos obligan a mirar-nos, a escuchar-nos y a unirnos con los otros y con lo otro. Sin vida no hay viaje y sin viajes/migraciones/desplazamientos la vida es sólo un concepto vacío, una palabra desprovista de fuerza y trascendencia. Los viajes a los que nos referimos son, literalmente, aquellas salidas que, ocasionalmente, programamos para visitar la playa, ir a la montaña, caminar por otras ciudades... Pero, si acudimos a la metáfora, los viajes son, también, acciones que emprendemos a diario para abandonar nuestra zona de comodidad y para aventurarnos a reconocer lo que está afuera de nosotros.

Estas dos vías de comprensión nos resultan útiles para hacer alusión a la tesis doctoral Artesanos de la salud y prácticas para el cuidado de la vida: aproximaciones a la medicina tradicional en el Norte de Antioquia (MÚNERA GÓMEZ, 2019), la cual fue desarrollada en el contexto del Doctorado en Ciencias Humanas y Sociales de la Universidad Nacional de Colombia, Sede Medellín. Acudimos a la imagen del viaje, pues, sin lugar a dudas, los ejercicios de construcción conceptual, de construcción metodológica y de construcción de las interpretaciones se dieron en el fragor de muchos desplazamientos, salidas y regresos.

La investigación doctoral surgió del interés de acercarnos a algunos hombres y mujeres sabedores o, en otros términos, a hombres y mujeres medicina, quienes habitan diferentes

1 La investigación que dio como resultado este artículo demandó el uso de varios consentimientos informados que fueron firmados por los ocho participantes de la misma. Además, durante todo el desarrollo del ejercicio de pesquisa, los participantes fueron conocedores de los avances y se vincularon a la exposición de las pinturas elaboradas por el artista invitado, además asistieron a la presentación final de las biografías comentadas, que el investigador construyó. veredas y municipios del Norte de Antioquia, Colombia, ${ }^{2}$ y quienes se han dedicado al desarrollo de acciones de salud comunitaria. Así pues, siguiendo la invitación de Constantino Cavafis (1982), emprendimos un largo viaje hacia muchas Ítacas, pusimos en el fuego algunas intenciones y nos dirigirnos a lugares insospechados "[...] a aprender, a aprender de sus sabios" (p. 51). El camino fue pródigo en enseñanzas y nos acercó a una comprensión de la vida humana a partir de los procesos de salud y enfermedad de las personas; además, nos permitió adentrarnos en algunas explicaciones del mundo y de las relaciones que en él se dan entre los diferentes seres que lo integran, a propósito del cuidado de la vida en sus diferentes manifestaciones. De este viaje, de las peripecias del caminar y de los aprendizajes logrados, queremos hablar en estas páginas.

El inicio de esta empresa estuvo marcado por el deseo de retornar a nuestras raíces para observar, escuchary dialogar. Queríamos llegar a donde los hombres y mujeres medicina -o abuelos sabedores como también los reconocimos- para recordar cómo aprendieron a preservar la vida, a partir de los pensamientos y las acciones que los integraron con la naturaleza, el cosmos y la divinidad. En concreto, pretendíamos profundizar en aquellas formas de salud que, a pesar del paso del tiempo, se mantenían como testimonio de una herencia de grupos étnicos que se asentaron en el Norte de Antioquia, tierra de los osos de anteojos, tierra de los nutabes, ${ }^{3}$ tierra de los abuelos

2 Colombia está integrado por treinta y dos departamentos, y una de estas divisiones es Antioquia. Este departamento, de acuerdo con la Ordenanza 41 del 30 de noviembre de 1975, se encuentra dividido, geográfica y administrativamente, en las siguientes regiones: Norte, Bajo Cauca, Magdalena Medio, Oriente, Suroeste, Nordeste, Valle de Aburrá, Occidente y Urabá. Al tiempo, estas regiones se encuentran subdividas en diferentes zonas, que están demarcadas por asuntos de orden geográfico. En estas regiones se agrupan los 125 municipios del departamento, sin embargo, hasta el momento, éstas son unidades de organización y administración, mas no gozan de ninguna independencia política o gubernamental.

3 Nutabaes o nutabes y tahamíes fueron las etnias indígenas que habitaron esta región; a la vez, osos de anteojos, imponentes y fieros animales, se encontraban aposentados en toda ella, en especial en la zona del Altiplano, en los tiempos 
participantes de la investigación.

Indagar por el cuidado era tarea necesaria, pues, reconocimos que, a pesar de la existencia de un modelo biomédico, que extendía sus formas de interpretación de la salud y la enfermedad por todos los puntos cardinales, los participantes de la investigación también disponían de otras formas de atención de la vida, que custodiaban con respeto y que desarrollaban con generosidad, sin caer en la mercantilización de un diagnóstico, una terapéutica o un producto. Los abuelos del Norte de Antioquia eran nuestros sabedores, así como también lo podían ser algunos integrantes de comunidades indígenas o comunidades negras. Pero, la opción fue por el pensamiento mestizo (CEBALLOS GÓMEZ, 2002), es decir, por los procesos de transculturación a los que se habían visto sometidos nuestros territorios después de los procesos de conquista y colonización, y que se veían materializados en formas de hacer concretas en el siglo XXI.

De esta manera, llegamos a diferentes municipios como San Pedro de los Milagros, Belmira, Entrerríos, Don Matías, Santa Rosa de

previos y paralelos a la llegada de los conquistadores a tierras americanas. Debido a las cruentas incursiones de los hombres que llegaron en barco desde el otro lado del Atlántico, tanto los indígenas, como los majestuosos animales desaparecieron de la zona, dejando prácticamente aniquilada la remembranza de su historia. De los osos, hoy por hoy, sólo se puede encontrar una escultura a la entrada del municipio de Santa Rosa de Osos. En cuanto a los indígenas, si bien no hay muchas claridades acerca de su desaparición, algunos documentos se atreven a plantear la idea, según la cual, varios de ellos se movieron hacia las márgenes del Mar Caribe, otros tantos murieron en el proceso de conquista, algunos más fueron usados como esclavos para la explotación de las minas de oro, mezclando así su sangre con la de los colonizadores, y una parte restante fue incluida en el resguardo indígena que tuvo lugar en las tierras de Ituango. A propósito de la llegada de los españoles y la conquista de los territorios, Escobar Villegas (2004, p. 58) asevera lo siguiente: "la llegada de los conquistadores europeos a la actual región de Antioquia tuvo lugar a principios del siglo XVI. Las expediciones se realizaron muy pronto en el período de las conquistas y los antiguos pueblos indígenas de esta región, como los nutabes, los tahamíes y los katíos, fueron vencidos rápidamente y sometidos luego a los tratamientos que la Corona española impuso a los indios en América: trabajos forzados, esclavitud, encierro en los resguardos, tributos obligatorios y mestizajes biológicos y culturales. La invasión de las tierras de los indios causó una notable disminución de la población en las sociedades aborígenes".
Osos y San José de la Montaña. Allí, gracias a la posibilidad del intercambio derivado de la palabra, nos dirigimos a veredas, corregimientos y barrios de los municipios para indagar por aquellas personas que llevaban a cabo ejercicios destinados a reestablecer la salud humana. Y, entre conversación y conversación, pudimos recordar que la diversidad de un país como Colombia y de un departamento como Antioquia no sólo estaba dada por la exuberancia de sus relieves con sus despliegues de fauna y flora, sino, también, por la fuerza y la magia de los seres humanos que los habitaban.

A medida que las visitas se hacían más frecuentes, nos llamó la atención el hecho de que estos abuelos, muchas veces sin pensarlo, ni desearlo, se convirtieron en custodios de la salud personal, familiar y comunitaria. De acuerdo con sus narrativas, ellos desempeñaban tareas asociadas al trabajo en el campo, tales como la agricultura y la ganadería; sin embargo, por distintos movimientos y azares de la vida, comenzaron a combinar sus desempeños de labriegos con el de agentes de los procesos de salud. Reconocer este asunto, en medio del trabajo de campo, nos llevó a plantear una pregunta central alrededor de la cual se comenzarían a tejer los subsiguientes encuentros y las conversaciones: ¿cómo el campesino deviene sanador? Con este norte claro, ya no sólo nos interesaba conocer diferentes prácticas de sanación, como las denominamos en un inicio, sino que, por el enfoque que le habíamos dado a la investigación, nos estábamos adentrando en las biografías y las subjetividades de hombres y mujeres guardianes de la salud.

Con esta reorientación, la investigación adquirió un horizonte concreto: profundizar en las sinuosidades del oficio de los campesinos sanadores desde su propia voz, trayectorias y recorridos. No eran todos los campesinos sanadores, dada la unidad contextual que ya aclaramos, nos enfocamos en los campesinos del Norte de Antioquia de las zonas de los Ríos Chico y Grande y, con ello, sus historias de vida se transformaron en el centro de nuestras con- 
versaciones para indagar sobre las terapéuticas y las formas de acompañamiento a quienes se hallaban con algún padecimiento. Entonces, el enfoque cambió, pues no nos adentramos en el tema de interés desde las prácticas de sanación. En lugar de ello lo hicimos a partir de los testimonios de vida, que establecían una fuerte conexión entre el pasado y el presente, para luego reconocer las prácticas para el cuidado de la vida, como las nombramos posteriormente, es decir, la herbolaria, el sobanderismo, la partería, la animería, la imposición de manos, la limpieza a través de la luz, la heliocromoterapia y la oración y la canalización de José Gregorio Hernández Cisneros.

En relación con lo anterior, en esta investigación tomamos algunas decisiones. Por un lado, como lo acabamos de señalar, volvimos a las experiencias y los testimonios de diferentes campesinos dedicados a cuidar de la salud a través de prácticas diversas, para ello recogimos sus narrativas $y$, con éstas, construimos otras narrativas mediadas por la literatura, la música y las pinturas. Al respecto, el hecho de optar por las voces de los campesinos como sanadores y agentes de la salud no desestimó las experiencias de algunos usuarios de las terapéuticas que aquéllos ofrecían, al contrario, en algunos casos, estas últimas fueron complementarias para la construcción de los relatos. De otro lado, no pretendimos construir un perfil epidemiológico de las enfermedades de la zona del Norte de Antioquia, no porque no fuera importante, sino porque más que clasificar las enfermedades, por el carácter interpretativo de la investigación, pretendíamos ahondar en los modos de comprender la salud y la enfermedad, como elementos constitutivos de la vida.

En trabajos subsiguientes se pueden llevar a cabo estos abordajes en diálogo con los elementos aportados por la biomedicina y los sistemas de gestión de salud, mas, para este trabajo en particular la finalidad fue otra, es decir, la de retomar las trayectorias de los sujetos y, desde sus voces, aportar a la construcción de un tejido intercultural de la salud y la compren- sión de todo sistema médico como un sistema cultural (LANGDON, 2014).

Otro de los elementos, que debemos destacar del presente trabajo, tiene que ver con que éste aportó al reconocimiento de formas de pluralismo terapéutico, que dieron lugar a procesos interculturales de diálogo de saberes allegados de la tierra, las plantas, los animales y los minerales; de conocimientos aportados por la biomedicina, y de elementos relacionados con la religión y la magia. De acuerdo con las comprensiones derivadas de esta investigación, este pluralismo terapéutico, que se evidenció a través de la coexistencia de prácticas de distinto orden, dio lugar al desarrollo de itinerarios terapéuticos (PERDIGUERO-GIL, 2006), a través de los cuales se puso en escena la capacidad de decisión de los agentes de salud y de los usuarios de los diferentes procedimientos curanderiles.

Por esta razón, en el trabajo dejamos de asumir a los campesinos sólo como una fuerza de producción, para reconocerlos como hijos de la tierra y como sujetos de conocimiento, con trayectorias personales y comunitarias, que ponían en relación las dimensiones humanas y las dimensiones sagradas. Desde esta consideración, esta investigación aportó elementos para desarrollar ejercicios que permitieron comprender al ser humano como totalidad holística, y para reconocer los diferentes campos en los cuales suceden los procesos de salud y enfermedad: el cuerpo, la mente, el espíritu y las emociones.

$\mathrm{Al}$ respecto, hemos de plantear que la voz, los saberes y las experiencias de los campesinos mestizos han estado por fuera de la construcción de una agenda intercultural de salud en un país como Colombia, pues, para nadie es un secreto que, a la hora de hablar de "medicina tradicional" lo primero que llega al pensamiento de algunas personas son las formas de curación, cuidado y tratamiento desarrolladas por comunidades indígenas o por comunidades negras. Pero, los campesinos a los que nos hemos referido no han sido vinculados 
a este tipo de medicina, ni a la consolidación de sistemas plurales de atención de la salud y la enfermedad, pues, según son vistos, éstos carecen de "ancestralidad" y "tradición”, y estas valoraciones, la mayor de las veces, sólo aplican para los otros grupos étnicos que mencionamos anteriormente.

Si para los indígenas y los negros ha sido difícil ser vinculados a este tejido intercultural, para los campesinos mestizos lo ha sido aún más, pues, ante la legislación colombiana, los territorios en los que éstos habitan no son reconocidos como espacios sagrados, que merecen ser delimitados como resguardos de la vida expresada en las plantas, los animales, los animales y, como tal, en la diversidad humana, salvo en el caso de algunos páramos. De ahí que, este trabajo propició el reconocimiento de los campesinos y de los vínculos que éstos establecían con las distintas formas de vida, que incidían en la sanación y el bienestar.

Para retomar el tema del diálogo, debemos aclarar que esta investigación se inscribió en un escenario de formación de carácter inter y trandisciplinar, por esta razón, el intercambio de perspectivas no sólo se dio entre formas y terapéuticas de las realidades socioculturales, sino que, en atención a la propuesta de formación del Doctorado en Ciencias Humanas y Sociales de la Universidad Nacional de Colombia, Sede Medellín. Es importante aclarar que retomamos los aportes de campos disciplinares como la Historia, la Antropología, la Antropología Médica, las Teorías del Discurso, entre otros.

Por último, esta investigación demostró que, en un país como Colombia, en el que después de la expedición de la Ley 100 de 1993 se intentó homogenizar todo el sistema de salud en atención a unas formas hegemónicas de entender y atender la enfermedad y la sanación de los seres humanos, aún podemos encontrar sistemas de cuidado de la vida, que se enraízan en las culturas y que beben de éstas para presentar diferentes modos de comprensión del ser humano, del cuerpo, el espíritu, la divinidad, en fin, de la salud como posibilidad de integración.

Estamos ante una investigación, que tomó distancia de lo establecido por la Ley 100 desde una mirada biomédica, que determinó formas únicas de atención de la salud en las regiones y las zonas que integran el país; en lugar de ello, nos acercamos a la enfermedad y la salud como procesos culturales, que ponen en escena las representaciones, los discursos y las prácticas de las comunidades y los sujetos que las integran. A través de esta investigación nos acercamos a un tipo de organización social y cultural, que por el poco reconocimiento que tiene ante la legislación colombiana, se ha mantenido por fuera de la construcción de una agenda intercultural para la comprensión de diferentes itinerarios terapéuticos.

Así pues, uno de los principales aportes de la pesquisa fue asumir que la salud es, en sí misma, un proceso artesanal (MÚNERA GÓMEZ, 2019), que implica la relación de tres dimensiones importantes: el hacer, representado en las manos; el decidir y el razonar, expresado en el pensamiento; y el establecer diferentes vínculos, lo cual se manifiesta a través del corazón. Desde esta óptica, se privilegió la voz de determinados sujetos de las comunidades que asumieron importantes tareas de atención de la salud, en relación o en tensión con los sistemas de la biomedicina. Ante la ausencia de unos determinados sistemas derivados de la medicina académica en las comunidades campesinas y ante el mosaico de culturas de la salud del que nos habla Hugo Portela (2015), reivindicamos las experiencias de hombres y mujeres que, sin pensarlo y, quizá, sin buscarlo, se adentraron en formas del cuidado integral.

\section{Sobre las metodologías} biográficas y narrativas: formas de hacer asociadas

\section{con la investigación}

Que las cosas escapen de sus formas, que las formas escapen de sus cosas y que vuelvan a unirse 
de otro modo. El mundo se repite demasiado. Es hora de fundar un mundo nuevo. (JUARROZ, 2012, p. 218-219).

Después de haber presentado el problema y el contexto de la pesquisa, es momento de explicitar algunos elementos acerca de la construcción metodológica, que pongan en evidencia las posibilidades y las formas a través de las cuales abordamos la pregunta orientadora de la investigación. En este momento de la escritura, recurriremos a la descripción y la narración de algunos de los acontecimientos más importantes, que nos posibilitaron el avance en los procesos de intercambio con las comunidades y los sujetos participantes. Cabe mencionar que, en esta parte del artículo presentaremos algunas comprensiones acerca de las metodologías biográficas y narrativas, la noción de perfiles de historia de vida y las estrategias para la recolección de la información a las que acudimos en este ejercicio de aproximación a la salud y la enfermedad, en comunidades campesinas, desde la perspectiva sociocultural.

Debemos aclarar que tomamos distancia de cualquier mirada instrumental del hacer en investigación y, más bien, nos acercamos a una idea de la metodología como espacio de "poiesis", ${ }^{4}$ es decir, como escenario de creación. Por tal motivo, reconocemos que la construcción metodológica no sólo se refirió a la determinación de unas relaciones entre enfoques, metodologías e instrumentos para la recolección de la información, sino que ésta implicó, también, otras acciones tales como: proposición del tema de investigación, construcción del estado del arte para situar el horizonte conceptual y comprensivo de la pesquisa, descripción de unas maneras de comprender el problema, elaboración de un núcleo de preguntas orientadoras y reconocimiento de algunos ámbitos conceptuales, para fundamentar las pretensiones investigativas.

Lo que haremos a continuación es articular

4 Esta noción es presentada por De Certeau (2000) como "una poiética". Él aclara que ésta viene de la voz griega poiein, y que tiene que ver con crear, inventar, generar. estos asuntos en clave de unos enfoques epistemológicos y metodológicos y, además, presentar las estrategias de investigación a partir de las cuales nos acercamos a los diferentes campesinos participantes y desarrollamos los procesos de intercambio, análisis y escritura, que nos ayudaron a destacar sus voces, experiencias y prácticas. ${ }^{5}$

Esta pesquisa se inscribió en una idea de investigación cualitativa, en tanto propiciamos un acercamiento a diferentes prácticas de carácter cultural asociadas a los procesos de salud y enfermedad, es decir, unas configuraciones, que se relacionaron con asuntos simbólicos y discursivos, y que derivan en procesos de interacción entre diferentes sujetos y sus comunidades. Por ello, retomamos la siguiente definición de María Eumelia Galeano (2007, p. 20), que nos permite aclarar cómo asumimos la perspectiva cualitativa en este ejercicio poiético: “[...] la investigación social cualitativa apunta a la comprensión de la realidad como resultado de un proceso histórico de construcción a partir de la lógica de los diversos actores sociales, con una mirada 'desde adentro', y rescatando la singularidad y las particularidades de los procesos sociales".

De este modo, nuestro interés fue acercarnos "desde adentro", esto es, desde la singularidad de las experiencias, los saberes y los procedimientos a formas de atención de los procesos de salud y enfermedad, llevadas a cabo por campesinos del Norte Antioquia, en particular de aquellos ubicados en municipios cercanos a la Vertiente de los ríos Chico y Grande: San Pedro de los Milagros, Don Matías, Belmira,

5 En la construcción de esta propuesta metodológica, ha sido muy importante el acercamiento a diferentes textos del profesor Orlando Fals Borda, en particular, a aquellos que abordan la idea de la investigación-acciónparticipación como posibilidad para acercarnos a las voces y las experiencias de los sujetos que, a lo largo de la historia, han sido silenciados o marginados. La propuesta de Fals Borda $(1979,1991,2009)$ reviste importancia para este trabajo, pues permite reconocer diferentes sujetos, prácticas y saberes que provienen de sectores subalternos -para el caso de los campesinos- $y$, desde aquí, la puesta en marcha de acciones que propicien el diálogo con múltiples racionalidades y formas de producir conocimiento. 
Entrerríos, Santa Rosa de Osos y San José de la Montaña. Como ya lo habíamos mencionado, la mirada se orientó hacia "los modos de hacer" de estos artesanos de la salud o, en otros términos, de estos hombres y mujeres medicina, ${ }^{6}$ y no a los efectos o los resultados que se pudieran evidenciar en los usuarios o en quienes se acercaran a ellos. Aunque hemos de aclarar que, en algunos momentos, esas otras narrativas también hicieron aportes a los procesos de análisis y comprensión de las prácticas, sin ser el objeto de interés de este estudio.

En este contexto, reconocimos las prácticas de atención a los procesos de salud y enfermedad como configuraciones culturales, que estaban fundamentadas en representaciones, discursos y saberes de los sujetos, tanto de los que las desarrollaban, como de sus usuarios. Así, desde una mirada cualitativa, nos acercamos a las formas de producción, circulación, apropiación y uso de los saberes sobre el cuidado de la vida y del cuerpo y, desde aquí, develamos la incidencia del simbolismo y el ritual en los procesos de curación. De nuevo, en palabras de Galeano, nos vinculamos a “[...] lo local, lo cotidiano, lo cultural para comprender la lógica y el significado que tienen los procesos sociales para los propios actores, que son quienes viven y producen la realidad sociocultural" (GALEANO, 2007, p. 20-21).

Para cumplir con lo anterior, nos fundamentamos en las metodologías biográficas y narrativas, a partir de la construcción de perfiles de historias de vida, pues no intentamos construir la "gran historia", que destacara todos los sucesos y los acontecimientos vividos por los campesinos sanadores, sino que nos interesó acercarnos a unas determinas perspectivas o dimensiones, asociadas con el devenir sanador. De aquí que acudimos a la noción de perfil de historia de vida, porque hubo unos

6 Con esta noción nos queremos referir a diferentes hombres y mujeres que han dedicado su vida a la medicina, es decir, curanderos tradicionales que han propiciado el conocimiento, el respeto y el rescate de diferentes tradiciones y saberes tradicionales de América. Esta noción puede ser ampliada en Tekpankalli $(1996,2005)$. determinados ángulos desde los cuales reconocimos y dialogamos con los participantes de la investigación. ${ }^{7}$

Al acercarnos a las metodologías biográficas y narrativas abordamos las experiencias y los recorridos, las peripecias y las comprensiones generadas por un sujeto frente a su propia vida. Aquí adquirieron importancia las redes de significado que los sujetos participantes lograban construir en sus relaciones con otros. Es por ello por lo que preferimos hablar del campo de las metodologías biográficas y narrativas como un escenario en el que se entretejen diversas formas del relato, tantísimas manifestaciones de la palabra y, en sí, de los géneros discursivos. El relato trae consigo la evocación de la experiencia, por ello, esta forma de la narración es otra posibilidad para vivir de nuevo la vida. Aquí se trata de reconocer, describir e interpretar las múltiples maneras de la subjetividad $\mathrm{y}$, en otros términos, las distintas trayectorias de los sujetos. Por esta razón, hablamos de microhistorias que, por lo general, no son reconocidas en la escritura de aquella gran historia que se condensa a través de una determinada hegemonía o fuerzas hegemónicas.

En este caso, de lo que se trató fue de elaborar una suerte de "biografía interpretada"18, en la que llevamos a cabo una reconstrucción de voces y testimonios y, a partir de la cual, pudimos hacer determinados énfasis en la misma forma de construir los relatos y en los modos de compartirlos. Por ello, al acogernos a la idea de perfiles de historia de vida, la tarea no se limitó a la recopilación de datos, más bien, nuestras acciones se encaminaron hacia un

7 Como bien lo expone Leonor Aurfuch (2010, p. 17), "la sola mención de lo 'biográfico' remite, en primera instancia, a un universo de géneros discursivos consagrados que tratan de aprehender la cualidad evanescente de la vida oponiendo, a la repetición abrumadora de los días, a los desfallecimientos de la memoria, el registro minucioso del acontecer, el relato de las vicisitudes o la nota fulgurante de la vivencia, capaz de iluminar el instante y la totalidad. Biografías, autobiografías, confesiones, memorias, diarios íntimos, correspondencias dan cuenta, desde hace poco más de dos siglos, de esa obsesión de dejar huellas, rastros, inscripciones, de ese énfasis en la singularidad que es a un tiempo la búsqueda de trascendencia". 
ejercicio comprensivo desde el tejido de voces, memorias y experiencias. Nos centramos, entonces, en determinados episodios de esa gran historia, que es la vida misma, para, por medio de ellos, adentrarnos en determinados argumentos y explicaciones de los participantes de la investigación.

Hemos de aclarar que, a través de las metodologías biográficas y narrativas es posible ingresar en las formaciones culturales para comprenderlas como escenarios en los cuales se produce conocimiento, y en las que se toman determinadas decisiones. A través de la construcción de una historia de vida se comprende un momento histórico determinado, no desde la voz hegemónica, mas sí desde la singularidad y el tejido de microhistorias y segmentos de historias. No obstante, a través de las narrativas y las historias de vida no sólo se da la recolección de datos sobre un momento histórico o una cultura particular, tal y como lo muestra Chase (2015); gracias al intercambio con los géneros narrativos, es posible adentrarse en un mundo de significados, acontecimientos y trayectorias de los sujetos, en otras palabras, es posible tener un mayor acercamiento a la subjetividad de los actores sociales.

En esta investigación, asumimos las narrativas en clave de las siguientes miradas: las narrativas como metodología de investigación, en tanto entregaron elementos epistemológicos y discursivos para fundamentar las pretensiones investigativas, así como para construir una red de significados compartidos alrededor del trabajo con la memoria y la experiencia; las narrativas como formas para orientar los análisis, pues iluminaron los caminos para construir los escenarios conversacionales con los participantes, recoger los datos entregados por ellos y desarrollar diferentes procesos interpretativos alrededor del núcleo de preguntas orientadoras del trabajo, y las narrativas como formas para orientar la escritura, pues aportaron en la construcción de otros relatos, que establecieron vínculos con la literatura, la música y el arte, a fin de configurar otras formas del discurso, en las que se destacaran los diálogos entre las voces de los diferentes participantes del trabajo.

En sintonía con lo dicho, nuestra apuesta epistemológica tuvo que ver con una defensa de las metodologías biográficas y narrativas como formas a través de las cuales es posible producir conocimiento y generar otras vías de comprensión del mundo de la diferencia y lo diferente. En este contexto, también sería una manera de agenciar el discurso académico o científico, ${ }^{8}$ que atiende a otras formas y estéticas de creación verbal o, como lo expresa Bajtin (1998), a otras formas de discurso.

$Y$ a fin de construir los perfiles de historia de vida, usamos algunas estrategias o formas de interacción tales como los relatos de experiencias, los círculos de palabra y la participación en terapéuticas y procedimientos de sanación, como caminos para aproximarnos a la memoria, las vivencias y las subjetividades propias de los sanadores. Apelar a los perfiles de historia de vida, como posibilidades narrativas, nos ayudó a tejer elementos alrededor de la pregunta orientadora de la investigación: ¿cuáles son los acontecimientos, las experiencias y las situaciones que hacen que un campesino devenga sanador y artesano de la salud? y, desde ella, pudimos comprender múltiples representaciones acerca del mundo, los territorios, las relaciones humanas y los vínculos con lo sagrado. Porque, además de introducirnos en las

8 Al respecto de esta idea, Jerome Bruner (1998, p. 46) destaca "dos modalidades de funcionamiento cognitivo, dos modalidades de pensamiento", las cuales aportan elementos específicos para construir y aprehender la realidad. La primera de éstas es la modalidad paradigmática o lógico-científica, que se fundamenta en la caracterización y la conceptualización, y en procedimientos tales como la explicación y la argumentación. La segunda es la modalidad narrativa, que guarda relación con la construcción de significados desde el uso de la metáfora y los tropos, con la descripción de la realidad desde la conciencia de los protagonistas de las historias y las experiencias, y desde la configuración de actos de habla, de carácter subjuntivo, que expresan deseos, exhortaciones, intercambio de posibilidades y prismas como formas de ver el mundo. Según Bruner, "[...] la modalidad narrativa permite llegar a conclusiones no sobre certidumbres en un mundo prístino, sino sobre las diversas perspectivas que pueden construirse para que la experiencia se vuelva comprensible". 
biografías y las subjetividades de los hombres y las mujeres participantes de la investigación, también intentamos destacar algunas particularidades de sus formas de pensamiento y de los territorios en los que viven.

Según Miguel Martínez Miguelez (2006, p. 220), "una historia de vida es una práctica de vida, una praxis de vida en la que las relaciones sociales del mundo que en esa praxis se da son internalizadas y personalizadas, hechas ideografía" y, en consonancia con este planteamiento, al volver sobre las biografías y las narrativas de sanadores, lo que hicimos fue orientar la mirada hacia sus propios recorridos vitales y a los modos a través de los cuales configuraron unas formas específicas de entender el cuerpo y de relacionarse con la enfermedad y la sanación. Se trató, en otras palabras, de hacer unos retratos "como acuarelas o apuntes a lápiz, perfiles más amplios como óleos y libros como modestos murales" (KRAUZE, 2013, p. 13), según la vida y los oficios de cada uno de los campesinos, como sujetos de saber que han vinculado otras epistemes y otros paradigmas a ese propósito de la búsqueda de la salud y del bienestar.

Las metodologías biográficas y narrativas propiciaron la construcción de los perfiles de historias de vida de los campesinos. ${ }^{9}$ Éstas partieron de la necesidad de propiciar otras formas de interacción con algunos sujetos de la cultura que, en muchos casos, no habían sido reconocidos por ciertas formas de conocimiento, instancias o instituciones de orden hegemónico. ${ }^{10}$ En esta dirección, a través de la escritura, construimos unos retratos que nos permitieron reconocer el rostro del otro, sus

9 De acuerdo con Fortunato Mallimaci y Verónica Giménez (2006), los métodos biográficos son unas de las principales tradiciones en el contexto de los abordajes cualitativos de la investigación social. Según estos autores, "los métodos biográficos describen, analizan e interpretan los hechos de la vida de una persona, para comprenderla en su singularidad o como parte de un grupo" (MALLIMACI; GIMÉNEZ BÉLIVEAU, 2006, p. 175).

10 Esta idea también podría leerse como sujetos que habían sido silenciados y cuyos saberes se habían visto subalternizados y marginalizados. Estas ideas se vieron enriquecidas con la lectura de Guha (2002). rasgos, pliegues y aquellas marcas distintivas de su experiencia como sanadores. Por ello, al amparo de estas metodologías, tomamos distancia de cualquier interés de cuantificar o establecer generalizaciones acerca del sistema de salud en Colombia o en la región Norte de Antioquia; mucho menos, construimos perfiles epidemiológicos de las comunidades y los municipios en los que tuvo asidero la investigación. En lugar de ello, a través de las estrategias para la recolección de la información, pudimos reconocer que, en las montañas del territorio antioqueño, habitan personas herederas de rituales y tradiciones, que son capaces de dialogar con las particularidades de una modernidad avasallante y, desde ahí, configuran otras políticas y estéticas de existencia.

De tal suerte, nos aproximamos a ocho artesanos de la salud, que desarrollaban prácticas para el cuidado de la vida. Tuvimos la oportunidad de conocer a campesinos que llevaban a cabo terapéuticas fundamentadas en la herbolaria o la yerbatería, es decir, que usaban las hierbas y las plantas medicinales para reestablecer funciones propias del cuerpo y generar procesos de armonización de las personas y los espacios en los que viven; también, establecimos vínculos con mujeres parteras, encargadas de acompañar procesos de gestación y nacimiento de seres humanos; igualmente, nos acercamos a formas de sobanderismo o compostura, a través del contacto con personas que "sobaban o componían" y propiciaban la articulación y el movimiento de zonas y partes del cuerpo afectadas por lesiones, caídas o traumatismos; finalmente, este ejercicio nos permitió tener proximidad a personas, que llevan a cabo un riguroso trabajo de acompañamiento espiritual, a través de la imposición de manos, la oración y la canalización de energías, entidades y divinidades.

A continuación, queremos destacar algunos elementos relacionados con los campesinos participantes de la investigación y con las prácticas para el cuidado de la vida que éstos desarrollaban: 
Esquema 1 - Información sobre los participantes de la investigación, las prácticas desarrolladas y sus vías de iniciación como sanadores

\begin{tabular}{|l|l|}
\hline $\begin{array}{l}\text { NOMBRE, EDAD Y UBICACIÓN } \\
\text { DEL SANADOR }\end{array}$ & PRÁCTICAS PARA EL CUIDADO DE LA VIDA \\
\hline $\begin{array}{l}\text { Berta Inés } \\
\text { Avendaño Agudelo }\end{array}$ & $\begin{array}{l}\text { Partería } \\
\text { Este oficio retoma los conocimientos de diferentes mujeres que se constitu- } \\
\text { yen en guardianas de la vida humana. En el caso de doña Berta, su iniciación } \\
\text { como partera fue el resultado de un asunto del azar, pues ella fue llamada } \\
\text { para asistir a la partera, que era considerada la "oficial". En el momento } \\
\text { del parto, se presentó el nacimiento de unos mellizos, según lo narró doña } \\
\text { Berta, el más grande de los dos no podía respirar. Ella recordó las palabras } \\
\text { de unas mujeres mayores, que decían que cuando los niños recién nacidos } \\
\text { no respiraban se les debía soplar duro por el ano para que les llegara aire a } \\
\text { los pulmones. Aunque no era la partera invitada, aplicó la instrucción que } \\
\text { había escuchado y le devolvió la vida al infante; de esta forma se aventuró } \\
\text { en el oficio de partera o comadrona como también se le conoce. }\end{array}$ \\
\hline
\end{tabular}

José Leonardo

Céspedes Rodríguez

76 años

San Pedro de los Milagros

Martha Genoveva

Osorno Restrepo

74 años

Don Matías

Argemira

Echeverry Molina

72 años

San Pedro de los Milagros

\section{Herbolaria}

Esta práctica se relaciona con el uso de variadas plantas, de carácter medicinal, que se usan en procedimientos de salud doméstica y comunitaria. Según lo narra don Leonardo, su iniciación como hombre conocedor de plantas se dio gracias a la herencia recibida de su familia. Desde niño observó cómo su padre usaba y preparaba diferentes bebidas, unturas o mezclas de plantas; además, su progenitor también se destacaba por ser componedor o sobandero. Gracias a los ejemplos reconocidos en el seno familiar, este hombre puso en práctica lo saberes aprendidos por las vías de la observación y del ejemplo.

\section{Heliocromoterapia}

Esta práctica para el cuidado de la vida implica la conjunción de tres fuentes de energía y sanación, a saber: el sol, el agua y el color. Doña Martha se inició en este oficio a partir de la lectura de un libro elaborado por Israel Rojas. Gracias a este texto, el aprendió que podía usar cuatro botellas de colores para energizar el agua con la ayuda del sol. Los siguientes son los colores y los usos de las botellas: azul para el equilibrio y las dolencias de orden mental, problemas de garganta y circulación; verde para las personas que tienen principios de cáncer o para las mujeres que tienen problemas en los ovarios; amarilla para personas con ataques epilépticos o con lepra; roja para hacer paños o masajes a personas parapléjicas. Además, esta sabedora comenzó a usar la botella transparente o blanca para preparar gotas para los ojos.

Además de este oficio, doña Martha también se especializó en la práctica de la herbolaria gracias a su sensibilidad y conexión con las plantas.

\section{Oración y canalización de José Gregorio Hernández Cisneros}

Esta forma de atención de la salud implica una relación con el espíritu de José Gregorio Hernández Cisneros, quien fue un médico venezolano que ejercía la medicina alopática o facultativa. Luego de la muerte del médico José Gregorio en Venezuela, a causa de un accidente automovilístico, comenzó a ser convocado por diferentes personas de las culturas populares, de esta forma la fe en su espíritu se hizo extensiva a diferentes regiones de Latinoamérica.

En el caso de doña Argemira, ella aprendió este oficio gracias a sus propios padecimientos de salud y los de su padre. En algún momento, ella pidió la ayuda de una mujer que también canalizaba el espíritu de este hombre. De esta manera, luego fue iniciada en las tareas terapéuticas. 


\begin{tabular}{|c|c|}
\hline $\begin{array}{l}\text { Blasina del Socorro } \\
\text { Mery de Correa } \\
62 \text { años } \\
\text { San José de la Montaña }\end{array}$ & $\begin{array}{l}\text { Sobanderismo } \\
\text { Esta es una práctica muy común en diferentes municipios del Norte de An- } \\
\text { tioquia, Colombia. Quienes la agencian ofrecen una atención de la estructu- } \\
\text { ra osteomuscular de los pacientes, la cual integra los músculos, los huesos, } \\
\text { los tendones o los cartílagos. } \\
\text { Tal y como lo cuenta doña Blasina, se inició en este oficio a partir de la ob- } \\
\text { servación de su abuelo, quien fue un médico en el municipio de Santa Rosa } \\
\text { de Osos. Esta mujer, en primera instancia, atendió a diferentes animales } \\
\text { domésticos y posteriormente extendió esta práctica a seres humanos. El } \\
\text { sobanderismo se puede agenciar por la vía de la oración con secreto o por } \\
\text { la del masaje con presión. }\end{array}$ \\
\hline $\begin{array}{l}\text { José David } \\
\text { Múnera Ochoa } \\
52 \text { años } \\
\text { San Pedro de los Milagros }\end{array}$ & $\begin{array}{l}\text { Limpieza a través de la luz } \\
\text { Don José David cuenta que comenzó a realizar esta práctica a partir del } \\
\text { seguimiento de su propia intuición. De acuerdo con los relatos y las obser- } \\
\text { vaciones del trabajo del sabedor, este oficio articula el uso de una fotografía } \\
\text { del ser consultante y de una vela. El terapeuta pone el material fotográ- } \\
\text { fico frente a la luz y, gracias a unas sombras que se dibujan en el cuerpo } \\
\text { de la foto, éste hace interpretaciones y construye diagnósticos. Luego, se } \\
\text { derrama parafina sobre las sombras para llevar a cabo la limpieza y para } \\
\text { desarrollar la sanación de padecimientos físicos, emocionales o mentales. }\end{array}$ \\
\hline $\begin{array}{l}\text { Ramiro Antonio } \\
\text { Velásquez } \\
49 \text { años } \\
\text { San José de la Montaña }\end{array}$ & $\begin{array}{l}\text { Imposición de manos } \\
\text { Esta práctica se articula con el aprovechamiento de la energía y la fuerza } \\
\text { que desprenden las manos en el contacto con plantas, animales y seres hu- } \\
\text { manos. Don Ramiro se inició en esta forma de atención física, espiritual y } \\
\text { mental gracias a un sueño en el que un hombre de rasgos similares a Jesús } \\
\text { de Nazareth le enseñó a imponer las manos para ayudar a los enfermos y } \\
\text { desvalidos. En otros contextos, esta práctica también se articula con el reiki } \\
\text { como forma de sanación con la ayuda de la energía universal. }\end{array}$ \\
\hline $\begin{array}{l}\text { Jesús María } \\
\text { Tobón Mazo } \\
44 \text { años } \\
\text { Belmira y Entrerríos }\end{array}$ & $\begin{array}{l}\text { Animería } \\
\text { Este oficio integra aspectos de la religiosidad popular del mundo católico. } \\
\text { Durante todas las noches del mes de noviembre, un hombre, que es llama- } \\
\text { do "el animero", se dirige al cementerio de su localidad para invitar a pasear } \\
\text { a las Ánimas del Purgatorio, para que vuelvan a los lugares que recorrieron } \\
\text { cuando eran seres humanos. A medida que el animero recorre las calles del } \\
\text { pueblo, pide a las personas que ofrezcan un padrenuestro por las almas, } \\
\text { para que éstas encuentren el descanso eterno. } \\
\text { Don Jesús María se hizo animero gracias a la observación de personas ma- } \\
\text { yores que, cuando él era más joven, desempeñaban esta tarea. Gracias a su } \\
\text { cercanía con la Iglesia Católica y al oficio de sepulturero, sintió una pro- } \\
\text { funda conexión con el culto de las Ánimas y, por esta razón, lo comenzó a } \\
\text { desarrollar. }\end{array}$ \\
\hline
\end{tabular}

Fuente: Elaborado por el autor de este artículo.

Vale la pena mencionar que no homologamos la metodología con el seguimiento de una serie de recetas o instrucciones para producir algo, sino que la asumimos como una ruta que fuimos transitando, y que tuvo incidencia en los modos de observar, escuchar, conversar, leer y escribir. Y para ampliar un poco más esta última idea, queremos introducir algunas descripciones y explicaciones acerca de cómo pudimos conocer a los participantes de la investigación y, gracias a esto, llevar a cabo las estrategias de recolección de información, las cuales, como ya lo mencionamos, aportaron en la elaboración de los perfiles de historia de vida y propiciaron el desarrollo de los análisis, que incluimos en este texto.

El principal criterio de participación, que planteamos para el desarrollo de la pesquisa, 
estuvo asociado con el acercamiento y la interacción con personas, de origen campesino, que desempeñaran oficios asociados a la atención de los procesos de salud y enfermedad, en diferentes municipios y veredas del Norte de Antioquia. No obstante, este criterio, que acabamos de exponer, nos hizo plantear otro más, el cual tuvo que ver con la necesidad de ir hasta los municipios, recorrer sus calles y caminos e indagar en las plazas, las veredas y los lugares de encuentro por aquellas personas que eran reconocidas como curanderos, sanadores, médicos tradicionales, entre otras formas de nominación.

Para ese momento de la pesquisa, que implicó el recorrido por los municipios y el reconocimiento de los posibles participantes, aún no teníamos claras las palabras más adecuadas para designarlos. Por eso, acudimos a diferentes nominaciones, pues aún no sabíamos cómo los reconocían las comunidades y, además, como se autopercibían ellos mismos. Lo que sí tuvimos claro, luego de la delimitación del contexto de la pesquisa, fue que intentaríamos llegar hasta las personas que, según las comunidades y sus integrantes, eran reconocidas por determinadas habilidades, capacidades o dones para sanar y ayudar a otros. De esta manera, paso a paso, fuimos tejiendo una red de personas y puntos de contacto, que nos ayudaron a llegar hasta los rincones y los parajes más insospechados de algunos municipios de la Meseta de los Osos, en el Norte de Antioquia. ${ }^{11}$

11 Como lo afirman, Mallimaci y Giménez Béliveau (2006, p. 177), al recurrir a las palabras de Smith, para la escritura de un relato de historia de vida "uno tiene que elegir un héroe o una heroína [...]. El investigador que recurre a la historia de vida no busca representatividad estadística, por lo tanto, el muestreo se basa en criterios de tipo teórico: en el muestreo selectivo, la persona se elige según rasgos considerados relevantes en términos conceptuales. Si nuestra investigación sigue un diseño multivocal o polifónico, lo importante será garantizar que nuestros entrevistados den cuenta de un rango amplio de experiencias individuales". En atención a lo anterior, en este trabajo, más que un asunto de elección, en este trabajo operó una construcción de criterios de participación, en los cuales los sujetos fueron convocados para tomar decisiones frente a la posibilidad de adentrarnos en sus relatos, experiencias e itinerarios de vida e itinerarios terapéuticos en cuanto a las formas de atención de la salud.
Cumplidos estos criterios, lo que hicimos fue generar la confianza suficiente para que aquellas personas, que nos habían mencionado en nuestros recorridos, nos permitieran ingresar hasta la intimidad de sus hogares o lugares de trabajo, para sostener algunas conversaciones acerca de sus recorridos vitales. Luego de conversaciones inesperadas, sellamos un acuerdo para vernos más seguido y, de este modo, permitir que las palabras se levantaran y comenzaran a distinguirse, como nos lo narra Nicolás Buenaventura (2007, p. 14), a fin de que se volvieran "árboles, enredaderas, arbustos y florecitas"; a fin de que se instauraran como remembranza de lo vivido y promesa de lo porvenir.

Las visitas se hicieron asiduas. Tal y como lo diseñamos, propusimos iniciar nuestras interacciones a través de unas entrevistas en profundidad,,$^{12}$ a partir de las cuales esperábamos acercarnos a los universos discursivos de los campesinos. Intentamos ser fieles a varias teorías sobre metodología de investigación y preparamos unos acercamientos paulatinos, que nos ayudaran a bordear los saberes y las prácticas, que queríamos conocer. Sin embargo, al llegar a los lugares en los que se encontraban los participantes, los escenarios discursivos se tornaron amplios y dieron lugar a conversaciones entre varias personas, lo que hizo que el protocolo de preguntas o tópicos de indagación fuera reevaluado y que, en su lugar, diéramos paso a intercambios más fluidos, en los que no sólo intervenimos el campesino y el "investigador", sino en los que también se vieron imbricados otros integrantes de la familia, vecinos y personas interesadas en escuchar las experiencias y los relatos de quienes se desempeñaban como guardianes de la salud.

Por lo anterior, las entrevistas en profundidad se resignificaron y convirtieron en círculos de palabra, es decir, en escenarios de conversación, cuya derivación fue un diálogo de saberes, a partir de la voz de un mayor. Gracias a lo ante-

12 Para ampliar algunas ideas acerca de la entrevista, sugerimos revisar Fontana y Frey (2015). 
rior, nos reunimos tres, cuatro o más personas, de muy diversas edades y, entre todos nosotros, circuló la palabra, tanto para preguntar, como para responder; tanto para afirmar, como para proponer otras cuestiones más. Así pues, en medio de los oficios cotidianos, conocimos esas historias que se escondían detrás de un rostro, una mirada y una sonrisa. El objetivo no estuvo puesto en el seguimiento cabal de un protocolo de preguntas, sino en la configuración de una conversación, que nos permitiera profundizar en muchas historias y avatares de unos sujetos campesinos, que alternaban sus tareas en el campo con el cuidado de la vida en sus múltiples manifestaciones. En muchas ocasiones, las preguntas y los comentarios de los otros participantes de los círculos de palabra implicaron aspectos que, desde la investigación, no habían sido contemplados y que resultaron de una importancia inusitada, para cumplir con las intencionalidades del trabajo.

Los círculos de palabra dieron lugar, entre otras cosas, a la socialización de múltiples relatos de experiencia de los campesinos, asociados a situaciones en las cuales sus prácticas habían sido importantes para la recuperación de la salud de algunas personas de sus comunidades o familias. Por esta razón, fueron otra importante estrategia para la recolección de la información, gracias a la cual pudimos develar representaciones, saberes y procedimientos alrededor de asuntos de sanación y enfermedad. El carácter de estos relatos fue oral, lo cual implicó la ampliación con gestos, onomatopeyas y hasta silencios, que ayudaban a marcar ciertos énfasis y transiciones en lo narrado.

En algunos casos, muchas de las preguntas o los comentarios hechos en las conversaciones permitieron la emergencia de los relatos, por medio de los cuales nos sumergimos en situaciones o anécdotas en las que se vio problematizada la efectividad o el éxito de algún procedimiento o terapia; del mismo modo, los relatos favorecieron la manifestación de sensaciones asociadas a las experiencias y los aprendizajes de los campesinos. Por este motivo, estas estrategias narrativas, que se desprendieron de los círculos de palabra, fueron importantes para describir, interpretar y, además, remembrar particularidades de las vivencias y reconocer en éstas la incidencia de la dimensión simbólica.

Al respecto, tal y como lo muestran Fortunato Mallimaci y Verónica Giménez Béliveau (2006, p. 175-176): "[...] estudiar el relato de los hechos de la vida de un individuo es el trabajo que un investigador realiza cuando recurre a la historia de vida. [...] tiene como elemento medular el análisis de la narración que este sujeto realiza sobre sus experiencias vitales".

Como una derivación de las anteriores estrategias de recolección de información, nos adentramos en la participación en terapéuticas y procedimientos de sanación. De acuerdo con la construcción del proyecto de investigación, consideramos la posibilidad de acudir a la observación participante ${ }^{13}$ como una vía para el levantamiento de la información requerida; empero, al estar en los encuentros y las conversaciones, ese rol del observador participante, que hace un registro de todo cuanto sucede $y$ lo guarda a través de algunos instrumentos de sistematización, dio paso a una implicación en desarrollo de las prácticas. En otras palabras, varios de los campesinos nos permitieron tener un mayor acercamiento a sus oficios para atender los procesos de salud y enfermedad, lo cual nos ayudó a reconocer cómo usaban diferentes materiales y recursos, y a vincularnos a lo que ellos mismos operacionalizaron.

Gracias a esta última estrategia, aquel rol del "investigador", que se fundamenta en la "asepsia” y la supuesta "objetividad", a través de construcción de unos protocolos de preguntas y formulaciones, dio paso a un compromiso con los procedimientos y al establecimiento de formas de cooperación. Incluso, en ciertos momentos, nosotros mismos nos convertimos

13 Para ampliar algunas consideraciones acerca de la observación participante y sus vínculos con la etnografía, recomendamos revisar las siguientes referencias: Angrosino (2012); Tedlock (2013) y Galeano (2007). 
en personas consultantes, pues tuvimos la posibilidad de exponer nuestras propias dolencias físicas, emocionales y mentales, así como algunas cuestiones de orden espiritual, que nos generaban inquietud. Lo anterior se vio animado por una sentencia pronunciada por varios de los campesinos: "Si quieren saber qué es lo que hago, primero lo deben conocer conmigo".

Sin embargo, debemos aclarar que esta estrategia, asociada con la participación en el desarrollo de las terapéuticas, sólo se dio en los casos en los que fue posible. Por cuestiones del azar y del tiempo, así como por asuntos relacionados con la edad o los desempeños de los campesinos, en ciertas ocasiones, no contamos con la posibilidad de asistir a una intervención determinada sobre un paciente o persona consultante, por lo cual sólo pudimos acercarnos a los oficios por medio de las descripciones y las narraciones recogidas en el trabajo de campo.

En el siguiente esquema, vamos a reunir los principales elementos de la ruta metodológica, que acabamos de exponer:

Esquema 2 - Relaciones entre enfoque, metodología y estrategias de investigación

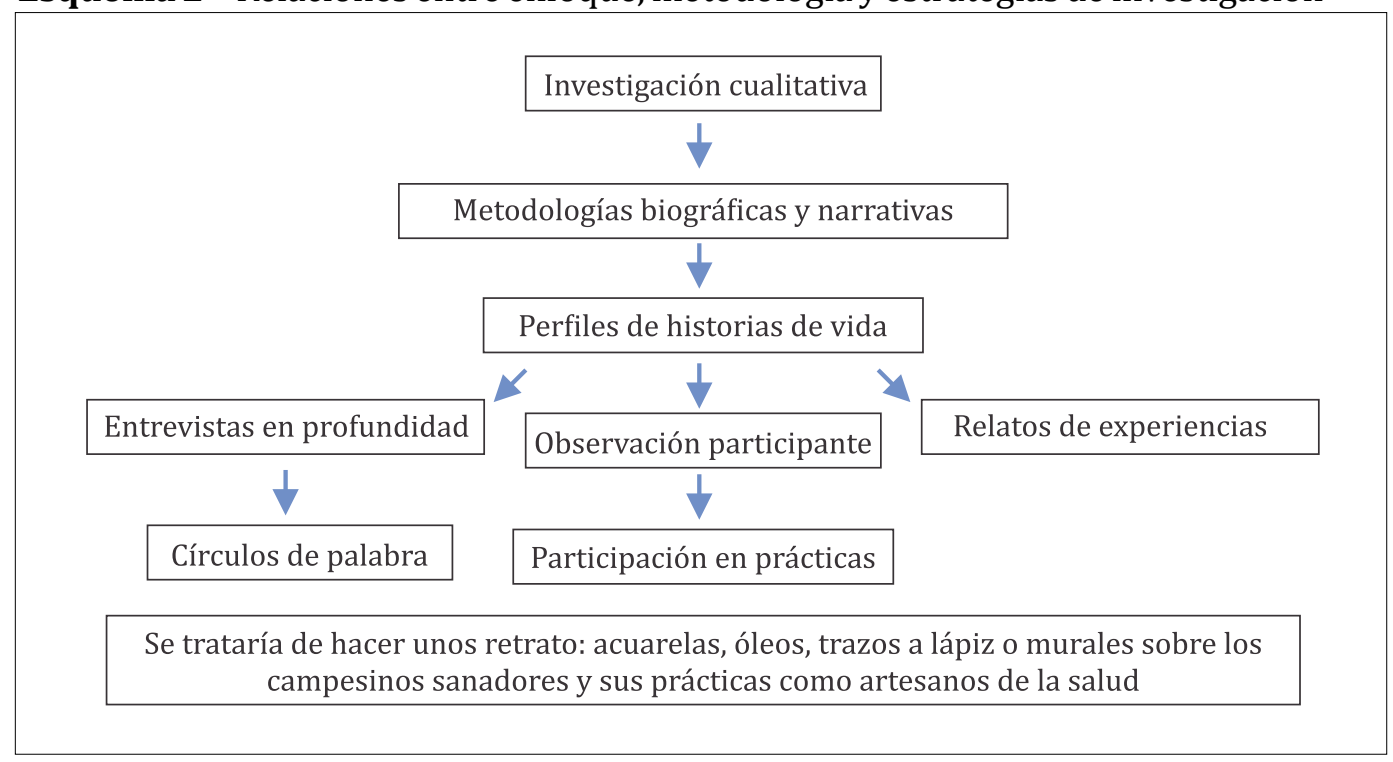

Fuente: Elaborado por el autor de este artículo.

Ahora bien, luego del desarrollo de las anteriores estrategias para la recolección de la información y de los correspondientes ejercicios de sistematización, codificación y proposición de líneas de análisis, llevamos a cabo la construcción de los perfiles de historias de vida de los participantes y la respectiva validación con ellos mismos. Aproximadamente, realizamos treintaicuatro encuentros con los campesinos, en los que, como lo acabamos de mostrar, ellos aportaron sus recuerdos y sus comprensiones a propósito de asuntos relacionados con algunas formas de la medicina tradicional en comunidades campesinas; posteriormente, como una manera para hacer devolución de lo interpretado, a través del análisis de los datos y la escritura de los perfiles, volvimos a las comunidades y a los participantes para leer los textos, a fin de conocer sus consideraciones y valoraciones frente a lo que allí expusimos.

Este nuevo encuentro nos permitió sostener otros intercambios discursivos, en los que los campesinos escucharon los perfiles de historia de vida, hicieron ampliaciones de las ideas allí reunidas y recomendaron algunos ajustes frente a los acontecimientos destacados. Igualmente, este momento de la devolución ayudó a materializar aquella metáfora propuesta por Krauze (2013), que ya compartimos líneas atrás, según la cual construir una historia de vida guarda proporciones con hacer el diseño de unos retratos o pinturas, que destacan los pliegues y los rasgos de los sujetos. Para tal fin, vinculamos al artista Carlos Antonio Aguilar 
Cardozo al trabajo de campo, para que nos apoyara en el propósito de construir algunos retratos de los campesinos, de los territorios en los que vivían y de las prácticas o las terapéuticas, que llevaban a cabo, para cuidar la vida.

En este caso, el acercamiento a las metodologías biográficas y narrativas, como formas para la construcción de saberes, también implicó al uso de otras formas de representación, que no quedaron circunscritas, únicamente, al lenguaje verbal, es decir, a las dimensiones orales o escritas. Gracias a la metáfora que ya destacamos, en el ejercicio investigativo, usamos la pintura como otra forma para construir las narrativas, y para acercarnos a aquellas experiencias y trayectorias de los sujetos participantes, las cuales fueron decisivas en aquel devenir sanador, que fue el eje de la investigación.

En estos encuentros finales del trabajo de campo, mientras hacíamos lectura de los perfiles de historia de vida y conversábamos sobre aquellas otras experiencias, que se desprendían de la investigación, Carlos Antonio, como artista, tomó algunos apuntes de los rostros de los campesinos, para luego, en su propio taller, instaurarlos como formas de representación, ${ }^{14}$ a partir de las cuales se daba lugar a otros énfasis en las historias de vida. Del mismo

14 A propósito de la noción de representación, Roger Chartier (2005, p. I) nos recuerda que éstas son "[...] las diferentes formas a través de las cuales las comunidades, partiendo de sus diferencias sociales y culturales, perciben y comprenden su sociedad y su propia historia". Igualmente, este historiador francés nos recuerda que las representaciones "[...] remiten a las modalidades específicas de su producción, comenzando por las intenciones que las habitan, hasta los destinatarios a quienes ellas apuntan, a los géneros en los cuales ellas se moldean. Descifrar las reglas que gobiernan las prácticas de la representación es pues una condición necesaria y previa a la comprehensión de la representación de dichas prácticas" (CHARTIER, 2005, p. VIII). Al respecto, acudimos al concepto de representación dada su riqueza para integrar diferentes formas de conocimiento y apropiación de los universos socioculturales. Este concepto es útil tanto para hacer referencia a las formas de pensamiento, como a los registros y los soportes en los que éstas se materializan. Así pues, volviendo a Chartier (2005), es muy potente la idea de pensar que estos procesos de percepción, interpretación y producción de la realidad se fundamentan en el reconocimiento de las diferencias de las comunidades, de allí que luego se desarrollen otros ejercicios de nominación, significación y establecimiento de relaciones. modo, los apuntes también retomaron aquellas particularidades de los territorios y de las formas del relieve, tan propias de una región como la del Norte antioqueño y, en particular, de la zona del Altiplano o la Meseta de los Osos. Finalmente, la lectura de los perfiles de historia de vida también le entregó algunos elementos al artista para que, en su taller, avanzara en la representación de aquellas terapéuticas, que los sujetos de la investigación aprendieron gracias a diferentes vías de iniciación.

El artista, entonces, escogió tres técnicas diferentes, para cumplir con aquel propósito de representar con otros códigos y símbolos la formación y la subjetividad de los artesanos de la salud, que se vincularon al trabajo. En este caso, él optó por los lápices de colores, las acuarelas y los óleos, como técnicas a través de las cuáles amplió los testimonios y los significados recogidos en el desarrollo de las estrategias investigativas. En total, se elaboraron veinticuatro pinturas durante la investigación, ocho por cada una de las técnicas antes mencionadas. A continuación, vamos a exponer algunas de las razones que justificaron la selección de cada una de estas técnicas, en atención a las ideas expuestas por Carlos Antonio en algunas conversaciones. Es importante destacar que, luego del trabajo de campo en los municipios de la investigación, sostuvimos algunas conversaciones con el artista para comprender, desde su lógica, algunos elementos de las obras que entregó y de las decisiones frente a las técnicas que seleccionó.

La primera técnica utilizada por el pintor fue la de lápices de colores. Gracias a ella el artista intentó detallar la esencia y la sensibilidad de los participantes, a través del énfasis en aspectos psicológicos, emocionales y en la conformación de los rostros de cada uno de ellos. El uso de las puntas de los lápices de colores fue útil para insinuar algunas cualidades, tales como la nobleza, la bondad, la introspección o la elocuencia, entre otras; además, se detallaron rasgos particulares tales como la forma de mirar, el modo de sonreír, la textura y el color de la piel y, en general, 
aquellas características que destacaban los pliegues de las biografías y las subjetividades de los sanadores. Los lápices de colores son muy utilizados para hacer bocetos, trazos y unos primeros diseños, que aportan, luego, al afloramiento de ideas, pues su uso exige un cuidado con los rasgos, así como un gran realismo en el momento de componer el retrato.

La segunda técnica a la que acudió el pintor, para intentar materializar la metáfora propuesta por Krauze (2013), fue la acuarela. El uso de esta resultó muy conveniente para destacar la composición paisajística de algunos territorios de la Meseta de los Osos, escenario que integra formaciones similares en cuanto al relieve se refiere. Una de las particularidades de la acuarela como técnica es que usa el agua como un material de sustantiva importancia, para la conformación de los trazos y las formas, y de este argumento se valió el artista para capturar diferentes imágenes de un territorio que es generador de agua y, por tanto, de vida.

A la par, dada la fluidez y la soltura que trae consigo esta técnica, con ella se detallaron diferentes fenómenos de la naturaleza, así como interrelaciones entre la tierra, el cielo, el agua, el aire y los animales. Las acuarelas, que resultaron del trabajo de campo, explicitaron algunas particularidades de las veredas y los municipios en los que vivían los participantes de la investigación, no obstante, al observarlas con cuidado se pueden identificar determinadas formas que se repiten o que mantienen rasgos similares, que comparten los municipios en los que se llevó a cabo la pesquisa.

El óleo fue la tercera técnica a la que acudió el artista, con ésta realizó un acercamiento a las prácticas o las terapéuticas que los agentes de la salud realizaban en sus veredas, corregimientos y municipios. Los óleos pintados en el contexto de la investigación lograron destacar diferentes componentes y dimensiones sutiles de los oficios dedicados a la preservación de la vida. Igualmente, gracias al uso de esta técnica fue posible poner en escena aquellas interrelaciones con la tierra, el cielo y lo sagrado, las cuales, según los testimonios de los participantes, se daban en sus tareas de servicio y ayuda hacia otros. Al respecto, es conveniente aclarar que, con los diferentes óleos, no se procuraba una determinada "fidelidad" o "realismo" a propósito de las prácticas para el cuidado de la vida; si bien, el origen de estas pinturas fueron los relatos y las descripciones de los sujetos que las agenciaban, el pintor le dio fuerza a otros elementos, cuyo reconocimiento no dependía de una condición sensorial, sino que se articulaba con otros componentes más sublimes y trascendentes.

No obstante, de acuerdo con las palabras del artista, el óleo es una técnica mixta que, en su caso, implicó una relación con las otras técnicas como lápices de colores, acuarela y acrílico, con el fin de generar ciertas texturas y apariencias propias de la pintura. De algún modo, esta técnica fue una especie de síntesis de otras formas creativas; además, al ser el último grupo de pinturas en realizar, permitió la recuperación de colores, formas y elementos, algunos de los cuales ya se habían presentado en las anteriores técnicas. Así, el artista empezó con los lápices de colores, de forma lenta y pausada, para elaborar los retratos de los rostros; posteriormente siguió con las acuarelas, para dar un acercamiento a los paisajes; y, luego de esto, acudió a los óleos y lugar a una irrupción de símbolos, formas y posibilidades creativas.

Para culminar esta reconstrucción de la memoria metodológica, es conveniente aclarar cómo llevamos a cabo la última parte del trabajo investigativo, luego de la escritura de los perfiles de historia de vida y de las pinturas. Dado que los campesinos, que hacían parte de aquella red dedicada al cuidado de la salud, no se conocían, decidimos llevar a cabo un encuentro de todos ellos, en la Universidad Nacional de Colombia, Sede Medellín. Para cumplir con este propósito, gracias a una sinergia entre la Dirección Académica, la Biblioteca Efe Gómez, la Facultad de Ciencias Humanas y Económicas de la Sede, pudimos llevar a cabo una exposi- 
ción de las veinticuatro pinturas elaboradas en el contexto de la pesquisa y, además, invitar a todos los participantes a un conversatorio, que tuvo lugar el día sábado 17 de febrero de 2018, alrededor de las pinturas exhibidas. Esta actividad favoreció el encuentro y el conocimiento de los campesinos; del mismo modo, fue un espacio para hacerles una devolución frente a sus aportes al desarrollo de la investigación; y, por último, permitió la vinculación de personas de la comunidad académica de la Universidad y de otras externas, para conversar con estos guardianes de la salud acerca de sus propios trayectos formativos, saberes y prácticas alrededor de las formas para cuidar la vida.

En este conversatorio, los participantes de la investigación enfatizaron en aquellos acontecimientos de su biografía sanadora, que fueron importantes para su devenir en artesanos de la salud. Al mismo tiempo, gracias a la posibilidad de la palabra, pudimos comprender que, a pesar de las distancias, entre las comunidades campesinas se mantienen determinadas formas de atención a la salud, que mantienen su correspondencia con la tierra, el agua, el aire, el sol y las divinidades, formas todas que nos hablan de una relación diferente con aquellos seres que nos rodean, incluso con la vida y con la muerte.

Lo dicho hasta el momento nos lleva a insistir en algo que afirmamos en páginas precedentes, esto es que nuestra forma de entender la metodología estuvo más asociada con el avance por una ruta rica en formas y relieves, y no tanto con el seguimiento de instrucciones o procedimientos previamente establecidos. La ruta transitada fue generosa en aprendizajes y estos nos permitió transformar las formas de mirar, hablar, investigar y escribir, por esta razón, comenzamos este texto recurriendo a las palabras de Roberto Juarroz (1991), pues reconocemos que es hora de comenzar a fundar un nuevo mundo, un mundo en el que los sujetos, los saberes y las prácticas establezcan otras relaciones y otras formas de hablar y de comprender lo humano.

\section{Unas palabras finales: la inminencia del cierre}

Optar por el desarrollo de metodologías biográficas y narrativas, en cualquiera de las esferas sociales, será siempre un llamado a la resistencia frente a los regímenes que pretenden regular y homogenizar las voluntades, las subjetividades y las formas de enunciación. Hoy en día, las ciencias, con el pretexto de objetivar y hacer neutral y transparente al conocimiento, someten a rigurosos procedimientos de asepsia el trabajo con sujetos y comunidades, de tal suerte, los investigadores renuncian incluso a su propia voz e instauran unos dispositivos de saber y de poder, para reducir a determinados códigos y nomenclaturas a los participantes de las investigaciones y a los testimonios que éstos aportan para el tejido de saberes.

De aquí la necesidad de adelantar trabajos en los que sea posible devolver la voz a los sujetos que, históricamente, han quedado por fuera de los circuitos de conocimiento del mundo ilustrado. Y, como vimos en las anteriores páginas, éste fue uno de los propósitos de la investigación doctoral Artesanos de la salud y prácticas para el cuidado de la vida: una aproximación a la medicina tradicional en el Norte de Antioquia (MÚNERA GÓMEZ, 2019). Gracias a este ejercicio de pesquisa fue posible ingresar a universos simbólicos en los que el diálogo entre trayectorias, saberes y prácticas campesinas para cuidar la vida se agenció de forma permanente.

En el ámbito de las ciencias humanas y sociales, es importante retornar a aquellos sujetos que hacen parte de las culturas populares y que tienen saberes que reflejan una construcción histórica de largo aliento. Así como nuestro pensamiento occidental cuenta con los aportes de innumerables pensadores, científicos y académicos, el mundo de la vida también se integra con la vida y el hacer de muchos sujetos que, de forma anónima, aportan saberes y prácticas, que resuelven situaciones concretas y que, sin lugar a dudas, mejoran la 
calidad de vida de las comunidades en ámbitos sociales, culturales, ambientales, educativos y, como el caso de la pesquisa de la que damos cuenta aquí, de la salud.

Le corresponde pues a la academia y al sistema mismo escolar retornar a aquellas formaciones humanas que agencian modos concretos de ser, estar y convivir. Este es quizá, el principal aporte de los enfoques y las metodologías biográficas y narrativas, que nos animan a mantener la esperanza y a reconocernos integrados a un todo gracias a la posibilidad de narrar y de tejer pequeñas historias que configuran el gran lienzo que nos hace humanos.

\section{REFERENCIAS}

ANGROSINO, Michael. Etnografía y observación participante en investigación cualitativa. Madrid: Morata, 2012.

ARFUCH, Leonor. El espacio biográfico. Dilemas de la subjetividad contemporánea. Buenos Aires: Fondo de Cultura Económica, 2010.

BAJTIN, Mijailovich. Estética de la creación verbal. México: Siglo XXI Editores, 1998.

BRUNER, Jerome Seymour. Realidad mental y mundos posibles: los actos de la imaginacion que dan sentido a la experiencia. Barcelona: Gedisa, 1998.

BUENAVENTURA, Nicolás. "Palabras". En: MAYA, Tita. Cuentos y pasatiempos. Bogota: Secretos para Contar, 2007.

CAVAFIS, Constantinos. Poesía completa. Madrid: Alianza Editorial, 1982.

CEBALLOS GÓMEZ, Diana Luz. "Quyen tal hace que tal pague". Sociedad y prácticas mágicas en el Nuevo Reino de Granada. Bogotá: Ministerio de Cultura, 2002.

CHARTIER, Roger. El mundo como representación. Estudios sobre historia cultural. Barcelona: Gedisa, 2005.

CHASE, Susan E. Investigación narrativa: multiplicidad de enfoques, perspectivas y voces. En: DENZIN, Norman K.; LINCOLN, Yvonna (coord.). Métodos de recolección y análisis de datos. Manual de investigación cualitativa volumen IV. Barcelona: Gedisa, 2015. p. 58-112.
DE CERTEAU, Michel. La invención de lo cotidiano. Tomo I. Las artes del hacer. México, D.F.: Instituto Tecnológico y de Estudios Superiores de Occidente, 2000.

ESCOBAR VILLEGAS, Juan Camilo. La historia de Antioquia, entre lo real y lo imaginario. Un acercamiento a la versión de las élites intelectuales del siglo XIX. Revista Universidad Eafit, v. 40, n. 134, p. 51-79,| abr./jun. 2004.

FALS BORDA, Orlando. El problema de cómo investigar la realidad para transformarla por la praxis. Bogotá: Tercer Mundo Editores, 1979.

FALS BORDA, Orlando. Acción y conocimiento: como romper el monopolio con investigación acción participativa. Santafé de Bogotá: CINEP, 1991.

FALS BORDA, Orlando. Una sociología sentipensante para América Latina. Bogotá: Siglo de Oro Editores/CLACSO, 2009.

FONTANA, Andrea; FREY, James. La entrevista. En: DENZIN, Norman; LINCOLN, Ivonna (coord.). Métodos de recolección y análisis de datos. Barcelona: Gedisa, 2015. p. 140-202.

GALEANO, María Eumelia. Estrategias de investigación social cualitativa. El giro en la mirada. Medellín: La Carreta Editores, 2007.

GUHA, Ranahit. Las voces de la historia y otros estudios subalternos. Barcelona: Crítica, 2002.

JUARROZ, Roberto. Poesía Vertical. Madrid: Cátedra, 2012.

KRAUZE, Enrique. El club de los biógrafos. En: BAZANT, Mílada (coord.). Biografía: modelos, métodos y enfoques. México: El Colegio Mexiquense, 2013. p. 11-15.

LANGDON, Esther Jean. La negociación de lo oculto: chamanismo, medicina y familia entre los Siona del bajo Putumayo. Popayán: Universidad del Cauca, 2014.

MALLIMACI, Fortunato; GIMÉNEZ BÉLIVEAU, Verónica. Historia de vida y métodos biográficos. En: VASILACHIS DE GIALDANO, Irene (coord.). Estrategias de investigación cualitativa. Barcelona: Gedisa, 2006. p. 175-212.

MARTÍNEZ MIGUELEZ, Miguel. Ciencia y arte en la metodología cualitativa. México: Trillas, 2006.

MÚNERA GÓMEZ, Mauricio. Artesanos de la salud y prácticas para el cuidado de la vida: aproximaciones a la medicina tradicional en el Norte de Antioquia. 2019. Tesis (Doctorado en Ciencias 
Humanas y Sociales) - Universidad Nacional de Colombia, Medellín, 2019.

PERDIGUERO-GIL, Enrique. Una reflexión sobre el pluralismo médico. En: JUÁREZ, Gerardo Fernández (coord.). Salud e interculturalidad en América Latina. Antropología de la salud y crítica intercultural. Quito: ABYA-YALA, 2006.

PORTELA, Hugo. Epistemes-otras: un desafío para la salud pública en Colombia. En: LANGDON, Esther; CARDOSO, Marina (org.). Saúde indígena. Políticas comparadas na América Latina. Florianópolis: UFSC, 2015. p. 145-168.

TEDLOCK, Barbara. La observación de la participa- ción y el surgimiento de la etnografía pública. En: DENZIN, Norman; LINCOLN, Ivonna (coord.). Las estrategias de investigación cualitativa. Barcelona: Gedisa, 2013. p. 198-227.

TEKPANKALLI, Aurelio. Una voz para los hijos de la tierra. Tradición oral del Camino Rojo. Chicago, P.S.: Graphics, 1996.

TEKPANKALLI, Aurelio. El regreso al camino de mis antepasados. Morelia: Impresos Hurtado, 2005.

Recebido em: 12/08/2019

Aprovado em: 12/11/2019

(cc) ErY-No é um artigo publicado em acesso aberto sob uma licença Creative Commons. 